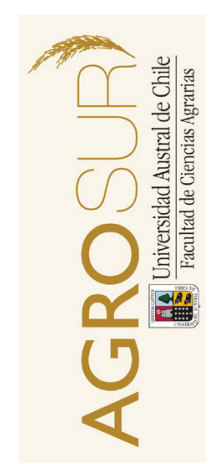

\section{Ocurrencia y distribución de residuos de plaguicidas en vegetales de hoja en el Cinturón Verde de Córdoba}

\author{
Incidence and distribution of pesticide residues \\ in leaf vegetables in the green belt of Cordoba
}

\author{
Faillaci, S.M. ${ }^{a *}$, Bastías-Montes, J.M. ${ }^{b *}$, Nassetta, M.M. ${ }^{a}$, Mangeaud, A. $^{a}$ \\ ${ }^{a}$ Universidad Nacional de Córdoba, Facultad de Ciencias Exactas, Físicas y Naturales - ICTA, \\ Av. Vélez Sarsfield 1611- X5016CGA - Ciudad Universitaria - Córdoba, Argentina. \\ ${ }^{b}$ Departamento de Ingeniería en Alimentos, Universidad del Bío-Bío, Av. Andrés Bello, 720, Chillán, Chile.
}

\begin{abstract}
A B S T R A C T
The objective of this study was to determine the presence of organophosphate and organochloride pesticides, and pyrethroid substances in the most consumed leaf vegetables in Cordoba, Argentina: Swiss chard (Beta vulgaris L. var. cicla L.), lettuce (Lactuca sativa L.), and spinach (Spinacia oleracea L.). The study covered the 2010-2013 period, and the geographic area included five vegetable-growing zones that make up the urban and periurban agriculture of Cordoba. The type of irrigation and the zones exceeding the maximum residue limits were considered. The "QuEChERS" Multiresidue technique was used to extract the pesticide residues from the plant tissue.

Residues were detected in 70 out of 311 analyzed samples. Pesticides were identified as endosulfan, deltamethrin, cypermethrin, bifenthrin, dimethoate, chlorpyrifos, chlorothalonil, malathion, and methamidophos. A total $22.5 \%$ samples were over the detection limit, while $10.92 \%$ exceeded the maximum residue limit. The Chi-squared test generated no positive associations between the presence of pesticides and a particular zone (Chi: 3.07; $\mathrm{p}=0.5467$ ). Among the vegetables, Swiss chard showed the highest amount of contaminants, although this difference was not significant (Chi: 2.81; $\mathrm{p}=0.2454$ ). Positive cases decreased in all the vegetables over the study period. Swiss chard decreased from $34.21 \%$ to $15.38 \%$, lettuce from 30.34 to $23.53 \%$; spinach started off with $17.86 \%$ and no positives were found in 2012 or 2013.
\end{abstract}

\title{
RESUMEN
}

El objetivo de este trabajo fue determinar la presencia de residuos de plaguicidas organofosforados, organoclorados y sustancias piretroides en los vegetales de hoja más consumidos en Córdoba-Argentina: acelga (Beta vulgaris L. var. cicla L.), lechuga (Lactuca sativa L.) y espinaca (Spinacia oleracea L.). El estudio abarcó el periodo 2010-2013 considerando la procedencia geográfica de la verdura de 5 zonas que componen la agricultura urbana y periurbana de Córdoba según el tipo de riego y cuáles de ellas excedieron los límites máximos residuales. Para la extracción de residuos de plaguicidas en tejido vegetal se utilizó la técnica de "QuEChERS” Multiresidue. Se detectó la presencia de residuos en 70 de las 311 muestras analizadas. Los plaguicidas identificados fueron: endosulfán, deltametrina, cipermetrina, bifentrina, dimetoato, clorpirifos, clorotalonil, malatión y metamidofos. El 22,5\% se identificó por encima de los Límites de Detección y 10,92\% de las muestras excedieron el Límite Máximo Residual. La prueba chi cuadrado no arrojó asociaciones positivas entre la presencia de plaguicidas y alguna zona en particular (Chi: 3,07; p=0,5467). Con respecto a la presencia sobre el tipo de verduras, la mayor cantidad de contaminantes se encontró en la acelga, aunque esa diferencia no fue significativa (Chi: 2,81; p= 0,2454). En todas las verduras ocurrió una disminución de los casos positivos encontrados a lo largo del tiempo del estudio. En el caso de la acelga, ésta disminuyó desde 34,21\% a 15,38\%; en lechuga de 30,34 a 23,53\% y en espinaca, comenzó con un 17,86\% y no se encontraron positivos en 2012 ni en 2013.

Palabras clave: contaminación, vegetales, límite máximo residual, pesticidas.

\section{INTRODUCCIÓN}

En los cinturones verdes de las ciudades conviven diferentes actividades y escalas productivas, entre ellas la actividad agrícola cuyos productos contribuyen, en mayor proporción, a la alimentación de la población de dichas ciudades. La mejora de la productividad muchas veces requiere de la utilización de agroquímicos y lo que es un insumo productivo para la mejora de las cosechas puede convertirse en contaminante predispo- 
niendo a potenciales daños en la salud humana. Estas prácticas en torno al uso de plaguicidas adoptadas por las quintas forman parte de una estrategia para conservar su posición (fuente de ingresos) y asegurar un producto de calidad que pueda alcanzar un buen precio durante el transcurso de la comercialización (Waisman, 2011). Sin embargo, a pesar de que los agroquímicos se han convertido durante el último siglo en un componente importante para incrementar los rendimientos de los cultivos y la producción de alimentos, también ha contribuido a la potencial presencia de sus residuos en los suelos, agua y aire originados a partir de su utilización masiva (Cooper y Dobson, 2007; Dhaliwal, 2009; Alexandratos et al., 2012). Durante las aplicaciones de los plaguicidas se han reportado muchos casos de intoxicación de agricultores, trabajadores rurales y sus familias. Cada año las intoxicaciones no intencionales matan a unas 355000 personas en todo el mundo, $y$ estas contaminaciones están asociadas a la exposición excesiva y su uso inapropiado (WHO, 2012; Alavanja, 2009; Alavanja y Bonner 2012). Los plaguicidas actúan por diferentes vías sobre los organismos. Los compuestos organoclorados (OC) (p. ej., aldrina, DDT, HCH, heptacloro, clordano, endosulfán) son en general insecticidas de contacto muy eficaces, y están estructuralmente relacionados con las hormonas esteroides y actúan sobre el respectivo receptor hormonal (Tebourbi et al., 2011). Los organofosforados (OF) (principalmente insecticidas, p. ej., paratión, malatión, clorpirifos, diazinón, diclorvos) actúan como inhibidores de la acetilcolinesterasa (AchE) mediante la interrupción de la transmisión del impulso nervioso a nivel de la sinapsis, mientras que los piretroides (insecticidas, por ejemplo, cipermetrina, deltametrina, bifentrina, esfevalerato) actúan sobre la regulación de los canales de sodio en las membranas celulares aumentando el flujo de iones. (Tomizawa y Casida 2005).

Los plaguicidas OC de primera generación fueron reportados tempranamente como ambientalmente persistentes, permaneciendo mucho tiempo en suelos y sedimentos y acumulándose en organismos no humanos con efectos tóxicos devastadores a nivel de población (Köhler y Triebskorn, 2013). Igualmente, también se transfieren en las cadenas alimentarias. El desarrollo de la resistencia de las plagas a estos productos químicos OC, instó a reemplazarlos por productos químicos nuevos y menos persistentes, como los OF, el carbamato y las sustancias piretroides (SP), supuestamente más específicos en la lucha contra las plagas (The Agrochemicals Handbook, 1991).

Para garantizar que estos residuos de contaminantes químicos no tengan efectos nocivos en la salud de la población, los organismos internacionales reconocidos como la Organización de las Naciones Unidas para la Agricultura y la Alimentación y la Organización Mundial de la Salud (FAO/OMS) a través de diferentes comi- tés de expertos dependientes o las autoridades sanitarias de diversos países establecen contenidos máximos permitidos de residuos en la gran mayoría de los alimentos que están basados en las cantidades residuales que aparecen tras un uso adecuado según las buenas prácticas agrícolas (BPA) (EFSA, 2008). Su empleo debe hacerse conforme a las normas de inocuidad las cuales establecen límites máximos admisibles de residuos (LMR) para minimizar el riesgo a su exposición o consumo. Sin embargo, ciertas prácticas agrícolas incorrectas hacen que se excedan esos límites, o bien que la presencia de varios residuos de un mismo producto (aunque las concentraciones estén por debajo de los valores aceptables), produzca un efecto aditivo (Pérez et al., 2009). Disminuir y reducir riesgos ha sido un reto permanente, ya sea para educar tanto a usuarios directos (productores y trabajadores) como a los consumidores de vegetales, frutas y sus productos (Fenik et al., 2011).

Con el fin de reducir la exposición ambiental de la población a estos productos químicos, los residuos tanto de las aplicaciones agrícolas, industriales como de las domésticas deben ser controladas estrechamente en el medio ambiente y en los alimentos (EFSA, 2016).

El cinturón verde de Córdoba (CVC), caracterizado por ser principalmente frutihortícola (producción de frutas, hortalizas pesadas y verduras livianas), requiere también del uso de agroquímicos que podría resultar un potencial riesgo tanto para el agricultor como para el consumidor si su concentración es elevada.

El principal objetivo de este trabajo fue el conocimiento de la ocurrencia de contaminantes organoclorados (OC), organofosforados (OF) y sustancias piretroides (SP) que pudieran estar presentes en el Cinturón Verde Córdoba (CVC), así como el conocimiento de sus niveles en la verdura liviana (VL), durante el periodo plurianual 2010-2013.

\section{MATERIALES Y MÉTODOS}

\section{Área de estudio}

El estudio se realizó en el Cinturón Verde de Córdoba, que abarca 5 zonas representativas según el tipo de riego (Figura 1):

- Zona 1: Río Primero o Suquía.

- Zona 2: Canal Maestro Sur.

- Zona 3: Canal Maestro Norte y sub-canales.

- Zona 4: Sistema de acequias.

- Zona 5: Río Segundo o Xanaes.

\section{Obtención y tratamiento de las verduras de hoja}

Durante febrero de 2010 a noviembre de 2013 se realizaron muestreos aleatorios en el Mercado de Abas- 


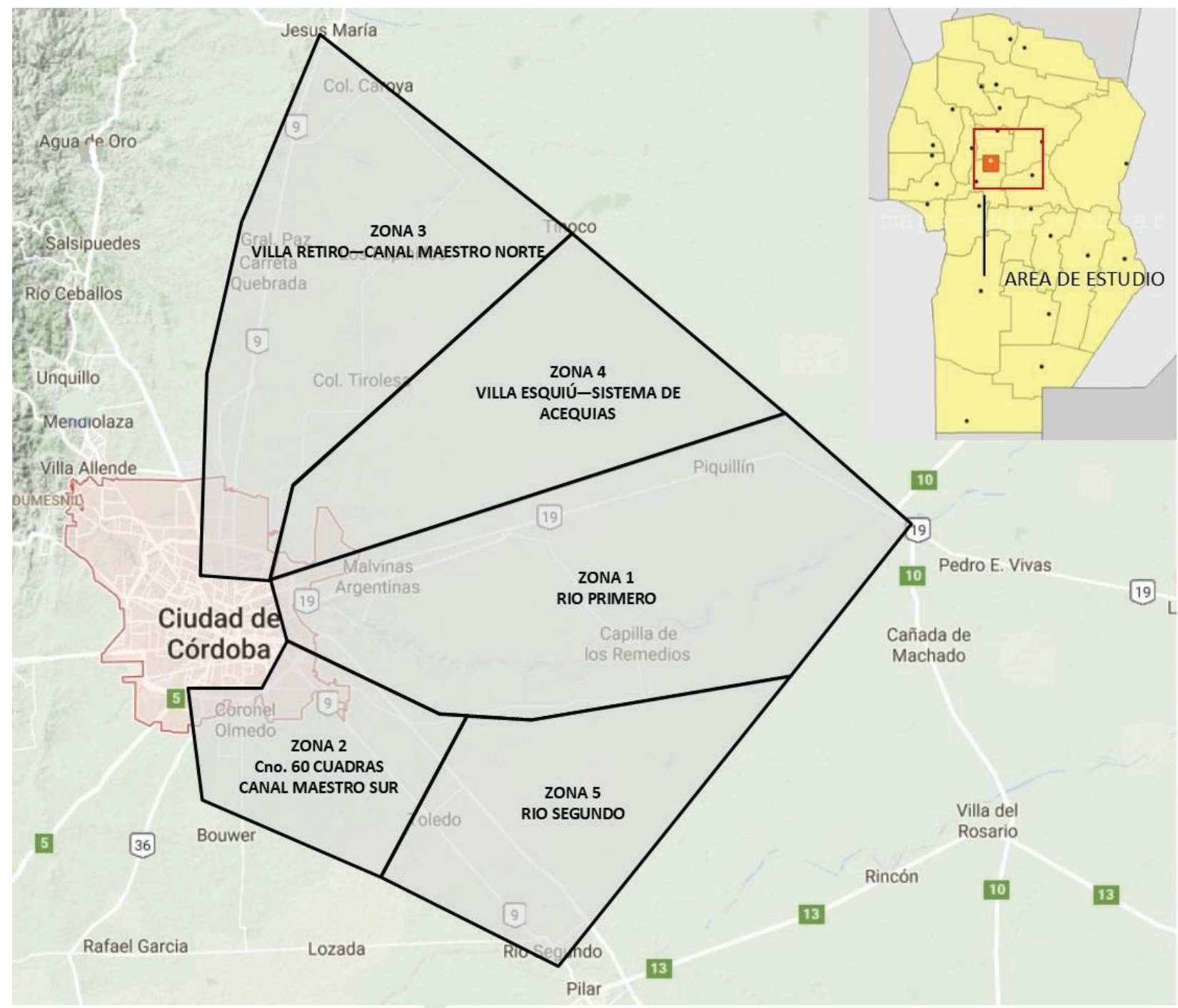

Figura 1. Agrupamiento de zonas según el tipo de riego del Cinturón Verde de Córdoba.

Figure 1. Arrangement of areas according to type of irrigation in the green belt of Cordoba.

to de la ciudad de Córdoba, se recolectaron muestras de las hortalizas de hoja: lechuga (Lactuca sativa L.), espinaca (Spinacia oleracea L.) y acelga (Beta vulgaris L. var. cicla L.) que son las más consumidas en la ciudad de Córdoba (GEACC, 2016). Sólo se muestrearon verduras de hoja cuyo origen era el cinturón verde de la ciudad de Córdoba y alrededores. Mediante datos corroborados por el Sistema de Control de Productos Frutihortícolas Frescos (SICOFHOR) instrumentado por el Servicio Nacional de Sanidad y Calidad Agroalimentaria de Argentina (SENASA) basado en el monitoreo, vigilancia y diagnóstico en frutas y hortalizas y la Dirección de Ferias y Mercados de la Municipalidad de Córdoba, se analizaron los compuestos organofosforados, organoclorados y sustancias piretroides provenientes de las verduras de hoja mencionadas.

\section{Muestras}

Un total de 311 muestras de $10 \mathrm{~kg}$ de material vegetal se tomaron al azar por triplicado cada una. Se las envasaron en bolsas de polietileno y fueron trasladadas al laboratorio a una temperatura de $4{ }^{\circ} \mathrm{C}$. Para la extracción de plaguicidas se utilizó la técnica "QuEChERS" (Quick, Easy, Cheap, Effective, Rugged, and Safe) (Anastassiades et al., 2003; Payá et al., 2007) que ha sido validada en el Laboratorio de contaminantes químicos del Instituto Nacional de Tecnología de Alimentos (INTA) de Castelar - Buenos Aires (Strada, 2014). Se determinó la concentración de los plaguicidas detectados y se consignó como ausente en el caso de que no hubiera detección. 


\section{Condiciones cromatográficas para el análisis de verduras de hoja}

La determinación de plaguicidas organoclorados y piretroides se realizó utilizando una cromatografía de gases Varian CG-ECD 3800 equipado con columna VF5ms $30 \mathrm{~m}, 0,25 \mathrm{~mm} \mathrm{ID}, 0.25 \mu \mathrm{m} \mathrm{df}$; inyector: $250^{\circ} \mathrm{C}$; detector: $300^{\circ} \mathrm{C}$; columna: $150{ }^{\circ} \mathrm{C} 4 \mathrm{~min}, 290^{\circ} \mathrm{C}$ rampa $8{ }^{\circ} \mathrm{C} / \mathrm{min} 30 \mathrm{~min}$.

La determinación de los plaguicidas organofosforados se realizó utilizando un cromatógrafo gaseoso Varian CG-PFPD 3800 equipado con columna SPB50 30 $\mathrm{m}, 0,25 \mathrm{~mm}$ ID, $0.25 \mu \mathrm{m} \mathrm{df}$; inyector: $250^{\circ} \mathrm{C}$; detector: $300^{\circ} \mathrm{C}$; columna: $80^{\circ} \mathrm{C} 1 \mathrm{~min}, 260^{\circ} \mathrm{C}$ rampa $20^{\circ} \mathrm{C} / \mathrm{min}$ $30 \mathrm{~min}$.

El límite de detección (LOD) fue establecido como la concentración más baja probada, lo que dio recuperación satisfactoria, precisión, relación señal/ruido e iones de diagnóstico de las abundancias relativas según los criterios de validación y control de calidad propuestos en las directrices europeas SANCO (EURL, 2007). Todos los análisis se realizaron en el CEPROCOR-Ministerio de Ciencia y Tecnología de Córdoba con técnicas acreditadas bajo ISO 17025:2005 y los resultados se expresaron en miligramos de plaguicida por kilogramo de muestra vegetal analizada $\left(\mathrm{mg} \mathrm{kg}^{-1}\right)$. Los residuos de plaguicidas bajo investigación y su límite de detección (LOD) de la matriz de muestra se enumeran en el Cuadro 1.

\section{Límites Máximos Residuales (LMR)}

Para el cálculo se tuvieron en cuenta aquellos residuos que fueron detectados o cuantificados al menos una vez durante el estudio. Los LMR tomados como referencia en este trabajo fueron el valor menor de un estudio comparativo entre Codex Alimentarius (que toma de referencia la legislación argentina) (Codex Alimentarius, 2017) y los establecidos en la Unión Europea (EFSA, 2008).

\section{Metodología estadística}

A los fines de asociar zonas, tipo de verdura con la presencia de los diferentes plaguicidas, se realizaron pruebas chi cuadrado de independencia (exactas). Para tal fin se utilizó el software R (R Core Team, 2016). El nivel de significación en todos los casos fue del 5\%.

\section{RESULTADOS Y DISCUSIÓN}

El estudio comprendió determinaciones realizadas en la verdura de hoja (acelga, lechuga y espinaca) que ingresaron en los días hábiles durante todo el año entre 2010 y 2013 al Mercado de Abasto de Córdoba. Estos productos provinieron de las quintas de la agricultura urbana y periurbana de la ciudad de Córdoba. Los valores encontrados se refirieron a 311 muestras totales. Los residuos de plaguicidas organoclorados (OC) detectados fueron el endosulfán y el clorotalonil; los organofosforados (OF): etil-clorpirifos, dimetoato, malatión y metamidofos, y las sustancias piretroides (SP) identificadas fueron bifentrina, cipermetrina y deltametrina. Del total, el 29,03\% de las muestras registraron presencia de compuestos totales $(\mathrm{OC}+\mathrm{OF}+\mathrm{SP})$ en el año $2010 ; 19,57 \%$ en $2011 ; 10 \%$ en 2012 y $20 \%$ en 2013. De las 311 muestras, 22,51\% de los casos presentó residuos de contaminantes y el 10,92\% registró violaciones al LMR (34 en 311). El más frecuente de los residuos detectados por encima del LOD fue el endosulfán $(9,32 \%)$, mientras que malation y metamidofos sólo se presentaron en una oportunidad cada uno $(0,32 \%)$ (Cuadro 2).

Cuadro 1. Análisis de sustancias activas y sus límites de detección ( $\left.\mathrm{mg} \mathrm{kg}^{-1}\right)$

Table 1. Active substance analysis and its limits of detection $\left(\mathrm{mg} \mathrm{kg}^{-1}\right)$.

Organofosforados acefato $(0,01)$; clorclorfenvinfos $(0,01)$; etil-cloropirifos $(0,01)$; metil-clorpirifos $(0,02)$; metil-demetona $(0,01)$; diazinon $(0,05)$; diclorvos - DDVP $(0,01)$; dimetoato $(0,01)$; disulfoton $(0,01)$; etil azinfos $(0,01)$; etion $(0,02)$; fenitrotión $(0,01)$; forato $(0,01)$; fosfamidona $(0,01)$; fosmet $(0,01)$; metamidofos $(0,01)$; metidación $(0,01)$; metilazinfos $(0,03)$; monocrotofos $(0,01)$, etilparación $(0,08)$, pirazofos $(0,01)$, metil-pirimifos $(0,02)$, quinalfos $(0,02)$, tiometón $(0,01)$, triclorfón $(0,01)$.

Organoclorados aldrín $(0,02)$; BHC - HCH $(0,01)$, captan $(0,08)$; alfa clordano $(0,02)$, gamma-chordane $(0,01)$; clorobencilo $(0,01)$; clortalonil $(0,01)$; dicofol $(0,02)$; dieldrín $(0,02)$; endosulfán $(0,01)$; endrina $(0,01)$; feraminol $(0,01)$; folpet $(0,03)$; $\operatorname{HCB}(0,01)$; heptacloro $(0,01)$; heptacloro epoxi $(0,01)$; hexaconazol $(0,01)$; imazalil $(0,02)$; iprodiona $(0,03)$; lindano $(0,006)$; metoxicloro $(0,01$; mclobutanil $(0,02$; mirex $(0,01)$, op'DDD $(0,007)$, op'DDE $(0,008)$, op'DDT $(0,01)$, penconazol $(0,01)$, procimidona $(0,01)$, pp'DDT $(0,02)$, quinometionato $(0,01)$, triadimefón $(0,01)$, vinclozolina $(0,01)$.

Piretroides bifentrina $(0,01)$; ciflutrina $(0,02)$; cipermetrina $(0,05)$; deltametrina $(0,01)$; fenpropatrina $(0,02)$; fenvalerato $(0,02)$; lamddtalotrina $(0,02)$; permetrina $(0,01)$. 
De los casos positivos, el endosulfán estuvo presente en el 41,43\%, y de ellos el 62,07\% (18 en 29) excedió el LMR, mientras que el otro OC detectado, el clorotalonil, sólo se detectó en la lechuga y representó un 2,86\% (2 de todos los casos positivos) de los plaguicidas que se detectaron en este cultivo. Al comparar estadísticamente las diferentes zonas y tomando en cuenta el porcentaje de muestras positivas por zona (Cuadro 3) se observó que en todas las zonas se presentó un porcentaje de casos positivos similar (entre 16,67\% y $31,25 \%)$. La prueba chi cuadrado no arrojó asociaciones positivas entre la presencia de plaguicidas y alguna zona en particular (Chi: 3,07; p=0,5467).

Con respecto a la presencia sobre el tipo de verduras, se observó una mayor cantidad de contaminantes en lechuga respecto a la acelga y la espinaca, pero esa

Cuadro 2. Contribución porcentual de residuos de cada contaminante por encima del Límite de Cuantificación y del Límite Máximo Residual

Table 2. Contribution in percentage of each pollutant residue above the Quantification Limit and Maximum Residual Limit.

\begin{tabular}{|c|c|c|c|c|c|c|c|c|c|c|c|}
\hline \multirow{2}{*}{$\begin{array}{l}\text { Residuo } \\
\text { de plaguicida }\end{array}$} & \multirow{2}{*}{ Tipo } & \multirow{2}{*}{$\begin{array}{c}\text { LOD } \\
\left(\mathrm{mg} \mathrm{kg}^{-1}\right)\end{array}$} & \multicolumn{3}{|c|}{ LMR (mg kg-1) } & \multicolumn{3}{|c|}{$\geq \mathrm{LOQ}$} & \multicolumn{3}{|c|}{$\geq$ LMR } \\
\hline & & & acelga & lechuga & espinaca & $\mathrm{n}$ & $\begin{array}{l}\% \text { del } \\
\text { total }\end{array}$ & $\begin{array}{c}\% \text { de los } \\
\text { positivos }\end{array}$ & $\mathrm{n}$ & $\begin{array}{l}\% \text { del } \\
\text { total }\end{array}$ & $\begin{array}{c}\% \text { de los } \\
\text { positivos }\end{array}$ \\
\hline bifentrina & SP & 0,01 & $0,05^{\mathrm{UE}}$ & $0,05^{\mathrm{UE}}$ & $0,05^{\mathrm{UE}}$ & 5 & 1,61 & 7,14 & 1 & 0,32 & 2,94 \\
\hline cipermetrina & SP & 0,05 & $0,7^{\mathrm{UE}}$ & $0,7^{\mathrm{CA}}$ & $0,7^{\mathrm{UE}}$ & 11 & 3,54 & 15,71 & 3 & 0,96 & 8,82 \\
\hline clorotalonil & OC & 0,01 & $0,01^{\mathrm{UE}}$ & $0,01^{\mathrm{UE}}$ & $0,01^{\mathrm{UE}}$ & 2 & 0,64 & 2,86 & 2 & 0,64 & 5,88 \\
\hline etil-clorpirifos & $\mathrm{OF}$ & 0,01 & $0,05^{\mathrm{UE}}$ & $0,05^{\mathrm{UE}}$ & $0,05^{\mathrm{UE}}$ & 5 & 1,61 & 7,14 & 4 & 1,29 & 11,76 \\
\hline deltametrina & SP & 0,01 & $0,5^{\mathrm{CA}}$ & $2,0^{\mathrm{CA}}$ & $2,0^{\mathrm{CA}}$ & 13 & 4,18 & 18,57 & 1 & 0,32 & 2,94 \\
\hline dimetoato & $\mathrm{OF}$ & 0,01 & $0,02^{\mathrm{UE}}$ & $0,02^{\mathrm{UE}}$ & $0,02^{\mathrm{UE}}$ & 3 & 0,96 & 4,29 & 3 & 0,96 & 8,82 \\
\hline endosulfán & OC & 0,01 & $0,05^{\mathrm{UE}}$ & $0,05^{\mathrm{UE}}$ & $0,05^{\mathrm{UE}}$ & 29 & 9,32 & 41,43 & 18 & 5,79 & 52,94 \\
\hline malatión & $\mathrm{OF}$ & 0,01 & $0,02^{\mathrm{UE}}$ & $0,5^{\text {UE }}$ & $3,0^{\mathrm{CA}}$ & 1 & 0,32 & 1,43 & 1 & 0,32 & 2,94 \\
\hline metamidofos & $\mathrm{OF}$ & 0,01 & $0,01^{\mathrm{UE}}$ & $0,01^{\mathrm{UE}}$ & $0,01^{\mathrm{UE}}$ & 1 & 0,32 & 1,43 & 1 & 0,32 & 2,94 \\
\hline Total positivos & & & & & & 70 & 22,51 & & 34 & 10,93 & \\
\hline Negativos $(<\mathrm{LD})$ & & & & & & 241 & 77,49 & & 277 & 89,07 & \\
\hline
\end{tabular}

Ref: LOD: Límite de Detección; UE LMR: Límite Máximo Residual de la Unión Europea, CA: Codex Alimentarius, JMPR: Reunión Conjunta FAO/ OMS sobre residuos de plaguicidas. LOQ: Límite de Cuantificación.

Ref: LOD: Detection Limit; EU MRL: Maximum Residue Limit of the European Union, CA: Codex Alimentarius, JMPR: Joint FAO/WHO Meeting on Pesticide Residues. LOQ: Quantification Limit.

Cuadro 3. Cantidad y frecuencia de casos por encima del LOD por zona de estudio.

Table 3. Number and frequency of cases above LOD by study area.

\begin{tabular}{|c|c|c|c|c|c|c|c|c|}
\hline \multirow[b]{2}{*}{ Zona } & \multicolumn{6}{|c|}{ Positivas } & \multicolumn{2}{|c|}{ Ausentes } \\
\hline & $\begin{array}{c}\text { Año } 2010 \\
(\mathrm{n})\end{array}$ & $\begin{array}{l}\text { Año } 2011 \\
\text { (n) }\end{array}$ & $\begin{array}{c}\text { Año } 2012 \\
\text { (n) }\end{array}$ & $\begin{array}{l}\text { Año } 2013 \\
\text { (n) }\end{array}$ & $\begin{array}{c}2010 / 2013 \\
(\mathrm{n})\end{array}$ & $\begin{array}{c}2010 / 2013 \\
\%\end{array}$ & $\begin{array}{c}2010 / 2013 \\
(\mathrm{n})\end{array}$ & $\begin{array}{c}2010 \text { / } 2013 \\
\%\end{array}$ \\
\hline 1 & 19 & 2 & 2 & 7 & 30 & 23,81 & 96 & 76,19 \\
\hline 2 & 3 & 0 & 0 & 0 & 3 & 16,67 & 15 & 83,33 \\
\hline 3 & 7 & 4 & 1 & 1 & 13 & 23,64 & 42 & 76,36 \\
\hline 4 & 8 & 1 & 3 & 2 & 14 & 17,50 & 66 & 82,50 \\
\hline 5 & 8 & 2 & 0 & 0 & 10 & 31,25 & 22 & 68,75 \\
\hline
\end{tabular}

Ref: LOD: Límite de Detección.

Ref: LOD: Detection Limit. 
diferencia no fue significativa (Chi: 2,81; $p=0,2454$ ). Igualmente, también pudo concluirse que en todas las verduras ocurrió una disminución de los casos positivos encontrados a lo largo del tiempo. En el caso de la acelga disminuyó de $34,21 \%$ a 15,38\%. En espinaca, comenzó con un 17,86\% y no se encontraron positivos en 2012 ni en 2013. Por su parte, la lechuga comenzó con 30,34\% y culminó en el 2013 con 23,53\% (Cuadro 4).

De los compuestos OF, el clorpirifos fue el más significativo aportando el 7,14\% de los contaminantes detectados en la lechuga donde el 80\% excedió el LMR (4 de las 5 muestras positivas). En menor orden se encontraron residuos de dimetoato, malatión y metamidofos, cuya presencia sólo se registró en 2010. Recientes estudios posteriores a éste realizados en el laboratorio del Mercado Central de Buenos Aires-Argentina, mostraron que la lechuga ocupaba el primer puesto de verduras con residuos de agroquímicos, en tercer lugar, la espinaca (15\%) y en sexto lugar la acelga (6\%) (INTA, 2016).

Los principales principios activos encontrados en Buenos Aires fueron clorpirifos, profenofos, metamidofos, dimetoato y carbofuran, entre otros, donde apreció un aumento de los "análisis de residuos excedidos" en un $5 \%$ en dos años (en promedio), con tendencia en aumento. Estos datos muestran una concordancia relativa con respecto a la presencia en los tipos de verduras afectadas del CVC.

Por último, y con respecto al tipo de VL se puede decir que el endosulfán significó el mayor residuo de contaminante detectado en todas las verduras durante el estudio ( $n=29)$ (Cuadro 2).

Estudios similares a éste fueron realizados sobre residuos de plaguicidas en la región de Galicia, España (González-Rodríguez et al., 2008) en 75 muestras de hortalizas de hoja verde (acelgas, espinacas y lechugas) donde encontraron 18 violaciones del LMR. Allí se observó que 15 de 18 no cumplían (agrupadas en "acelgas+espinacas") vs 3 de18 provenientes de lechugas. El $24 \%$ de las muestras analizadas no cumplían con las regulaciones, pero solo un $4 \%$ de lechugas contra un $20 \%$ de acelgas y espinacas, mientras que en la provincia de Buenos Aires - Argentina fueron las lechu- gas entre las hortalizas de hoja que presentaron una mayor tasa de contaminación (Szczesny, 2014). Esto puede deberse a las lechugas son altamente sensibles a las plagas y necesitan aplicaciones sucesivas de los tratamientos con pesticidas, dejando en consecuencia altos niveles de residuos que son tolerados.

En este estudio se ha demostrado que lechuga y acelga prácticamente han presentado plaguicidas en proporciones similares $(22,46 \%$ vs $27,16 \%$, respectivamente).

El trabajo "Plaguicidas en el territorio de la provincia de Buenos Aires, información toxicológica, ecotoxicológica y comportamiento ambiental" - de características similares a la de Córdoba- (OPDS, 2013), señala a la cipermetrina, el clorpirifos, la deltametrina, el dimetoato, el endosulfán y el metamidofos como los plaguicidas más frecuentemente encontrados (85\%). Éste advierte la presencia de residuos de clorpirifos, metamidofos y dimetoato (entre otros) al igual que los hallados en la VL del CVC, destacando que se trata de compuestos de "banda roja" (toxicidad aguda).

La prevalencia de endosulfán en este estudio se asemeja a los resultados obtenidos en los estudios realizados en el Banco Alimentario de la ciudad de La Plata - Argentina que indicaron que el $77 \%$ de las muestras analizadas $(n=47)$ dieron resultados por encima del LMR, siendo los endosulfanes los de mayor frecuencia de detección (43\%) seguido por etil-clorpirifos (Ronco, 2015). Éste último compuesto fue el principal OF detectado en el periodo de estudio se ubicó en el cuarto lugar de los contaminantes totales $7 \%$ de los casos positivos) mientras que, entre las SP, la deltametrina y la cipermetrina se presentaron en proporciones casi similares $(18,57 \%$ y $15,71 \%$, respectivamente), y la bifentrina en menor medida $(7,14 \%)$ como se observa en el Cuadro 2. Idénticos compuestos (SP) analizados en verduras livianas por la autoridad sanitaria en Valencia, España, en el periodo 2010-2012 muestran la presencia de residuos de cipermetrina, bifentina y deltametrina $(0,6 ; 1,3$ y $1,3 \%$, respectivamente); mientras que el clorotalonil se destacó entre los compuestos OC $(7,1 \%)$ y etil-clorpirifos entre los OF $(20,5 \%)$ como

Cuadro 4. Comparación de contaminantes presentes por año en acelga, lechuga y espinaca.

Table 4. Comparison of contaminants per year in Swiss chard, lettuce and spinach.

\begin{tabular}{|c|c|c|c|c|c|c|c|c|c|}
\hline \multirow{2}{*}{ Verdura } & \multicolumn{2}{|c|}{2010} & \multicolumn{2}{|c|}{2011} & \multicolumn{2}{|c|}{2012} & \multicolumn{2}{|c|}{2013} & \multirow{2}{*}{ Promedio } \\
\hline & $\mathrm{n}$ & $\%$ & $\mathrm{n}$ & $\%$ & $\mathrm{n}$ & $\%$ & $\mathrm{n}$ & $\%$ & \\
\hline acelga & 13 & 34,21 & 5 & 35,71 & 2 & 12,5 & 2 & 15,38 & 27,16 \\
\hline espinaca & 5 & 17,86 & 1 & 16,67 & 0 & 0 & 0 & 0 & 13,95 \\
\hline lechuga & 27 & 30,34 & 3 & 11,54 & 4 & 10,53 & 8 & 23,53 & 22,46 \\
\hline Total & 45 & 29,03 & 9 & 19,57 & 6 & 10 & 10 & 20 & 22,51 \\
\hline
\end{tabular}


los de mayor proporción (Pardo-Marín et al. 2015). Claramente, el número de muestras con residuos de SP son 10 veces menores que los niveles obtenidos en este estudio, no así el uso de etil-clorpirifos que manifiesta una presencia relativa 3 veces superior al CVC. Sin embargo, cualquiera de ellos, resultan sumamente elevados si se los compara con estudios realizados en Madrid, España en 2010-2011 donde el 32\% de las verduras analizadas (152 productos vegetales diferentes) presentó residuos detectables donde el número de muestras que excedió el LMR no superó 1\% (CarreteroGómez et al., 2014).

Como se señaló más arriba, la cipermetrina es la segunda de los casos positivos detectados de las SP, mientras estudios realizados por González-Rodríguez et al. (2008) en Ourense al NE de España señalan que es la cipermetrina el insecticida que mayor número de positivos por encima del LMR en verdura de hojas, al igual de lo informado por la organización no gubernamental PANNA (Pesticide Action Network North America) que recoge los datos corrientes de la USDA's Pesticide Data Program (PAN Database, 2017), donde resaltan los hallazgos de cipermetrina muy por encima del resto de plaguicidas $(21 \%$ y $1 \%$, respectivamente).

\section{CONCLUSIONES}

Se detectó la presencia de residuos de plaguicidas organoclorados: endosulfán y clorotalonil; plaguicidas organofosforados: etil-clorpirifos, dimetoato, malatión y metamidofos; y sustancias piretroides: bifentrina, cipermetrina y deltametina en la verdura liviana proveniente del Cinturón Verde de Córdoba (CVC) en el periodo plurianual 2010-2013. El 22,51\% de los casos (70 de 311) presentó residuos de contaminantes y el 10,93\% (34 de 311) registró violaciones al LMR. La definición de zonas en base a los cursos de riego facilitó la comprensión de las prácticas agrícolas que se llevan a cabo en el CVC, aunque no se detectaron diferencias significativas de la presencia de residuos de plaguicidas entre zonas.

Los resultados sugieren que los agricultores del Cinturón Verde de Córdoba deberían ser capacitados en el manejo adecuado de agroquímicos.

\section{Agradecimientos}

- Subsidio SECyT - Universidad Nacional de Córdoba, Argentina. 2012-2014. Uso de plaguicidas organoclorados y organofosforados en las aguas de riego utilizadas en la agricultura periurbana del cinturón verde de la ciudad de Córdoba. Res. 162/12.

- Dirección de Ferias y Mercados de la Municipalidad de Córdoba.

- Grupo de Investigación Universidad del Bío-Bío GI 172122/VC.Final del formulario.

\section{REFERENCIAS}

Alexandratos, N., Bruinsma, J., 2012. World agriculture towards 2030/2050: the 2012 revision. ESA Working paper. No. 12-03. FAO, Rome. http://www.fao.org/docrep/016/ap106e/ap106e.pdf

Alavanja, M.C.R., 2009. Pesticides use and exposure extensive worldwide. Reviews on Environmental Health 24, 303309.

Alavanja, M.C.R., Bonner, M.R., 2012. Occupational pesticide exposures and cancer risk: a review. Journal of Toxicology and Environmental Health, Part B 15, 238-263. https://doi.org/10.1080/10937404.2012.632358

Anastassiades, M., Maštovská, K., Lehotay, S.J., 2003. Evaluation of analyte protectants to improve gas chromatographic analysis of pesticides. Journal of Chromatography A, 1015(1-2), 163-184. https://doi.org/10.1016/ S0021-9673(03)01208-1

Carretero-Gómez, M.M., Iñigo-Núñez, S., Sánchez-Pérez, E., 2014. Resultados del control de residuos en alimentos 2010-2011. Documentos Técnicos de Higiene y Seguridad Alimentaria no 13. Programa de Control y Vigilancia de Alimentos. Comunidad de Madrid, España.

Codex Alimentarius, 2017. Maximum Residue Limits (MRLs). http://www.fao.org/fao-who-codexalimentarius / codex-texts/maximum-residue-limits/en/ (acceso, 01.05.2017).

Cooper, J., Dobson, H., 2007. The benefits of pesticides to mankind and the environment. Crop Protection 26, 1337 1348. https://doi.org/10.1016/j.cropro.2007.03.022

Dhaliwal, G.S., 2009. An Outline of Entomology. Kalyani Publishers, New Delhi, India.

European Food Safety Authority (EFSA), 2008. Scientific Opinion of the Panel on Plant Protection Products and their Residues (PPR Panel) to evaluate the suitability of existing methodologies and, if appropriate, the identification of new approaches to assess cumulative and synergistic risks from pesticides to human health with a view to set MRLs for those pesticides in the frame of Regulation (EC) 396/2005. EFSA Journal 704, 1-85. http://dx.doi.org/10.2903/j.efsa.2008.705

European Food Safety Authority (EFSA), 2016. The 2014 European Union report on pesticide residues in food. EFSA Journal 14(10), 4611. http://dx.doi.org/10.2903/j. efsa.2016.4611

European References Laboratories (EURL), 2007 Method Validation \& Quality Control Procedures for Pesticide Residues Analysis in Food \& Feed Document No. SANCO/2007/3131. http://www.crl-pesticides.eu/library/ docs/allcrl/AqcGuidance_Sanco_2007_3131.pdf

Fenik, J., Tankiewicz, M., Biziuk, M., 2011. Properties and determination of pesticides in fruits and vegetables. Trends in Analytical Chemistry 30(6), 814-826. https:// doi.org/10.1016/j.trac.2011.02.008

Grupo de Epidemiología Ambiental del Cáncer en Córdoba (GEACC), 2016. Valoración de la exposición a plaguicidas en cultivos extensivos de la Argentina y su potencial impacto sobre la salud. Facultad de Ciencias Médicas de la Universidad Nacional de Córdoba, Argentina. FONCyT-PICT 2008-1814.

González-Rodríguez, R.M., Rial-Otero, R., Cancho-Grande, B., 
Simal-Gándara, J., 2008. Occurrence of fungicide and insecticide residues in trade samples of leafy vegetables. Food Chemistry 107(3), 1342-1347. https://doi. org/10.1016/j.foodchem.2007.09.045

Instituto Nacional de Tecnología Agropecuaria (INTA), 2016. Uso de agroquímicos: se presentaron datos contundentes. https://inta.gob.ar/noticias/uso-de-agroquimicos-se-presentaron-datos-contundentes (acceso, 20.03.2017).

International Organization for Standardization (ISO), 17025:2005. General requirements for the competence of testing and calibration laboratories. https://www.iso. org/standard/39883.html

Köhler, H.R., Triebskorn, R., 2013. Wildlife ecotoxicology of pesticides: can we track effects to the population level and beyond? Science 341, 759-765. https://doi. org/10.1126/science.1237591

Organismo Provincial para el Desarrollo Sostenible (OPDS), 2013. Plaguicidas en el territorio bonaerense: información toxicológica, ecotoxicológica y comportamiento ambiental. Dirección Provincial de Recursos Naturales, Programa Gestión Ambiental en Agroecosistemas. Buenos Aires, Argentina.

Pardo-Marín, O., Marín-Villuendas, S., Gallart i González, J., 2015. Vigilancia de residuos de plaguicidas en productos vegetales en mercado. Niveles y evaluación de la exposición en la Comunitat Valenciana (2010-2012). Generalitat Valenciana. Conselleria de Sanitat Universal i Salut Pública. Valencia, España.

Payá, P., Anastassiades, M., Mack, D., Sigalova, I., Tasdelen, B., Oliva, J., Barba, A., 2007. Analysis of pesticide residues using the Quick Easy Cheap Effective Rugged and Safe (QuEChERS) pesticide multiresidue method in combination with gas and liquid chromatography and tandem mass spectrometric detection. Analytical and Bioanalytical Chemistry 389(6), 1697-1714. https://doi. org/10.1007/s00216-007-1610-7

Pesticide Action Network North America (PANNA), 2017. Database. http://www.pesticideinfo.org/Index.html (acceso, 20.03.2017).

Pérez, M., Segura, A., García, R., Colinas, T., Pérez, M., Vázquez, A., Navarro, H., 2009. Residuos de plaguicidas organo- fosforados en cabezuela de brócoli (Brassica oleracea) determinados por cromatografía de gases. Revista Internacional de Contaminación Ambiental 25, 103-110.

R Core Team, 2016. R: A language and environment for statistical computing. R Foundation for Statistical Computing, Vienna, Austria.

Ronco, A., 2015. Algunas respuestas sobre los impactos del uso de plaguicidas para el control de plagas en agroecosistemas de la Región Pampeana. Ciencia e Investigación. 65(2), 63-71.

Strada, J., 2014. Evaluación del nivel de residuos de plaguicidas en granos de cereales y oleaginosas aplicados en el campo y en almacenamiento en la región central de Argentina. Tesis Doctoral, Universidad Nacional de Córdoba. 224 p.

Szczesny, A., 2014. Producción hortícola bajo cubierta (compilado). Ciudad Autónoma de Buenos Aires. Ediciones INTA, Buenos Aires, Argentina.

Tebourbi, O., Sakly, M., Rhouma, K.B., 2011. Molecular Mechanisms of Pesticide Toxicity. In: Stoytcheva, M. (Ed.), Agricultural and Biological Sciences. Pesticides in the Modern World - Pests Control and Pesticides. Exposure and Toxicity Assessment. InTech Europe, Rijeka, Croatia, pp. 297-332. https://cdn.intechopen.com/pdfswm/20786.pdf

The Agrochemicals Handbook, 1991. Kidd, H., James, D.R. (Eds.), Royal Society of Chemistry, Information Services, Cambridge, England. https://trove.nla.gov.au/version/25285953 (acceso, 30.10.2017).

Tomizawa, M., Casida, J.E., 2005. Neonicotinoid insecticide toxicology: mechanisms of selective action. Annual Review of Pharmacology and Toxicology 45, 247-268. https://doi.org/10.1146/annurev.pharmtox.45.120403.095930

Waisman, M.A., 2011. Superando dualismos: trayectorias socio-productivas en el abordaje de las transformaciones en la estructura social hortícola platense. Universidad Nacional de La Plata, Mundo Agrario 2011, 12(23).

World Health Organization (WHO), 2012. The WHO recommended classification of pesticides by hazard and guidelines to classification. World Health Organization, Geneva, Switzerland. 